\title{
Peripapillary circle of Zinn-Haller revealed by fundus fluorescein angiography
}

\author{
Myung-Kyoo Ko, Dong-Seob Kim, Yoon-Koo Ahn
}

\begin{abstract}
Aims-To observe the vascular pattern of the peripapillary circle of Zinn-Haller in humans by fundus fluorescein angiography.

Methods-307 cases (from 212 patients) of fundus fluorescein angiograms performed in patients with myopic degeneration were evaluated to find the circle of Zinn-Haller and to observe its fundus fluorescein angiographic features.

Results-15 cases (from 13 patients) with the circle of Zinn-Haller were found. It appeared as concentric or zigzag-shaped vascular fillings within the temporal crescent region. All cases were observed in pathological myopia with peripapillary atrophy and a tilted disc. Each arterial circle showed variations in location and shape.

Conclusions-The temporal part of the circle of Zinn-Haller can be revealed by fundus fluorescein angiography particularly in pathological eyes with prominent peripapillary atrophy and a tilted disc. The morphological variation of this arterial circle should be considered.

(Br F Ophthalmol 1997;81:663-667)
\end{abstract}

The blood supply of the optic nerve head and peripapillary choroid has been studied by many investigators using various techniques, but the precise microvascular anatomy is still controversial. $^{1-8}$ However, it has been the consistent opinion that the short posterior ciliary arteries (SPCAs) are major contributors supplying the optic nerve head..$^{2-58}$ SPCAs have a variable origin from the ophthalmic

Accepted for publication 10 April 1997 artery within the orbit. Some SPCAs course without branching through the sclera directly into the choroid; others divide within the sclera to provide branches to both the choroid and the optic nerve. Often, branches from the SPCAs form a complete or incomplete circle within the sclera surrounding the optic nerve: this arterial anastomosis is the so called circle of Zinn-Haller $(\mathrm{CZH})$. Although the existence of an arterial circle has been questioned, ${ }^{2} 459$ many reports have described the existence of this arterial circle and its role in supplying the optic nerve head and peripapillary choroid. $^{6-810-13}$

It has been the general consensus that the intrascleral $\mathrm{CZH}$ cannot be appreciated by fundus fluorescein angiography (FFA) because of the blocking effect of retinal pigment epithelium, choroid, and sclera. However, as the choroidal vessel can be seen clearly in pathological conditions such as high myopia or ocular albinism, the intrascleral $\mathrm{CZH}$ can be observed by FFA through the thin sclera, especially when it is accompanied by a tilted disc. To our knowledge, there have been no reports describing the angiographic appearance of the $\mathrm{CZH}$, so we conducted a retrospective investigation of FFAs performed in patients with myopic degeneration.

\section{Materials and methods}

We evaluated retrospectively the medical records and FFAs of 212 patients (307 eyes) with pathological myopia, seen at the eye clinic of Hanyang University Hospital from March 1984 until April 1996. Of these 212 patients, 123 were male and 89 were female. Ages ranged from 13 to 72 years, with a mean of 39.8 years. All patients underwent colour and red-free fundus photography, and FFA using a standard technique. Fundus photographs and fluorescein angiograms for each patient were reviewed by two of us (MKK, DSK).

Vascular filling patterns of the peripapillary region, especially the temporal peripapillary region, were reviewed. Angiographic evidence of the $\mathrm{CZH}$ was based on the shape, filling time, continuity, and, most importantly, location. The $\mathrm{CZH}$ was represented as the definite concentric or zigzag-shaped continuous vascular filling located between the optic disc margin and the temporal crescent margin. The angiographic features of the $\mathrm{CZH}$, including location, shape, and presence or absence of the entry point of the temporal paraoptic SPCA, were studied. We excluded all cases which were remarkably out of focus and with equivocal continuity of vascular filling.

$\mathrm{SE}=$ spherical equivalent $\mathrm{D}=$ dioptres; $\mathrm{TPSPCA}=$ temporal paraoptic short posterior ciliary artery; + = present; $-=$ absent 

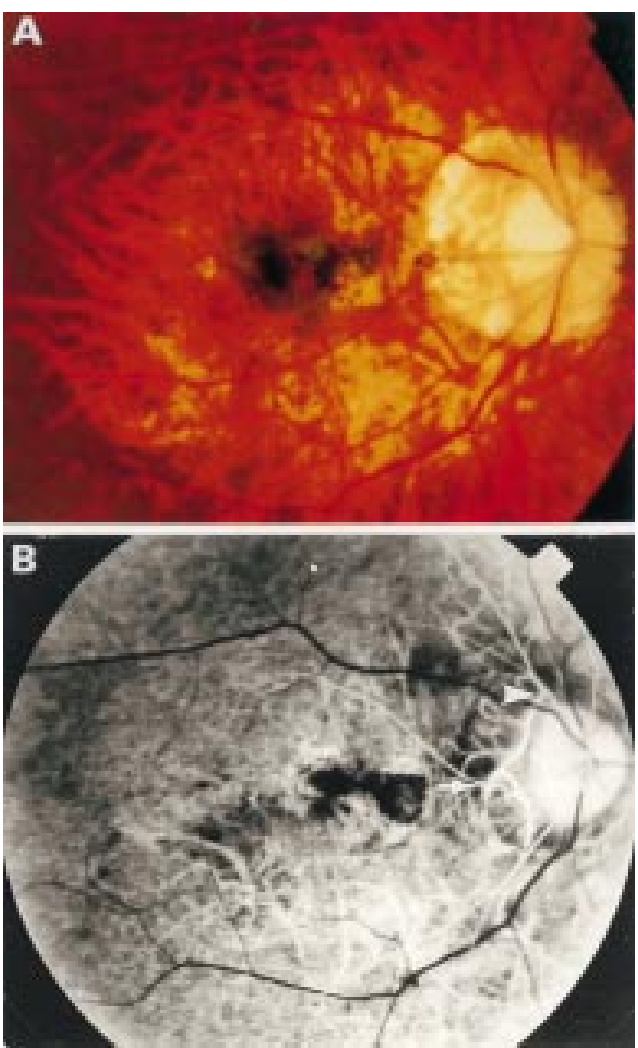

Figure 1 (A) Fundus photograph of the right eye of patient 1 with a refraction of -18.0 dioptres shows the concentric circle of Zinn-Haller near the temporal disc margin. (B) Arterial phase of fundus fluorescein angiography shows the temporal part of the circle of Zinn-Haller (between arrowheads) within the temporal crescent. Note the entry point of the temporal paraoptic short posterior ciliary artery (arrow) which forms the upper and lower vascular arcades.

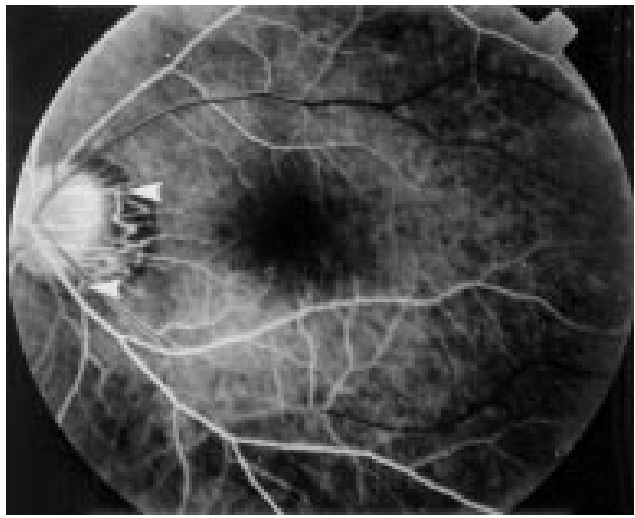

Figure 2 The left eye of patient 2 with a refraction of -7.0 dioptres. The arteriovenous phase of fundus fluorescein angiography shows the zigzag shape of the temporal part of the circle of Zinn-Haller (between arrowheads).

\section{Results}

Of the 307 eyes from 212 patients, the temporal part of the $\mathrm{CZH}$ was found in 15 eyes $(5 \%)$ from 13 patients $(6 \%)$. FFA revealed the $\mathrm{CZH}$ bilaterally in two patients. The refractive error in the 15 cases ranged from -7.0 dioptres to -22.0 dioptres (mean 14.13 (SD 4.80) dioptres). Table 1 gives the clinical information and the angiographic features of each case with the $\mathrm{CZH}$. All cases were from patients with severe myopic degeneration and tilted disc.

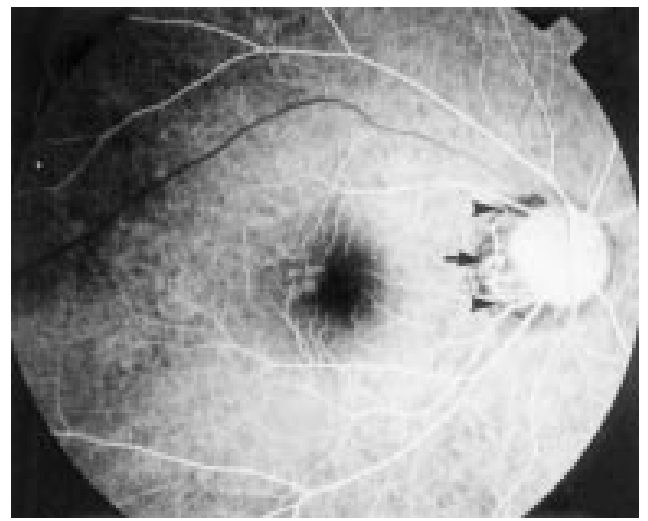

Figure 3 The right eye of patient 3 with a refraction of -12.5 dioptres. The arteriovenous phase of fundus fluorescein angiography shows the temporal part of the circle of Zinn-Haller (between arrowheads) within the temporal crescent. Note the entry point of the lateral paraoptic short posterior ciliary artery (arrow).

The complete circle could not be observed by FFA, and we evaluated mainly the temporal part of the circle. The fundus fluorescein angiographic features of the $\mathrm{CZH}$ were as follows:

(1) It was located within the temporal crescent region although the variability in location relative to the optic disc could be observed (Figs 1-10).

(2) It appeared as a concentric vascular filling in $10(67 \%)$ cases (Figs 1, 3-8) and as a zigzag-shaped vascular filling in five $(33 \%)$ cases (Figs 2, 9, 10) during the choroidal or arterial phase of FFA.

(3) The entry point of the temporal paraoptic SPCA from which the $\mathrm{CZH}$ might be divided into upper and lower arcades could be observed in six (40\%) cases (Figs $1,3,5,7,8)$, whereas it was equivocal or absent in nine $(60 \%)$ cases (Figs 2, 4, 6, 9, 10). The appearance of the entry point also showed variation, so it appeared near the optic disc margin (Figs 1, 3, 7, 8) or even beyond the border of the temporal crescent (Fig 5).

(4) It was a continuous vascular filling without interruption so far as we could see (Figs 1-10).

\section{Discussion}

It is well known that in myopic degeneration progressive chorioretinal stretching and atrophy produce a temporal crescent in which white sclera can be seen. If the scleral thinning and tilted disc, which may cause changes in the arrangement of the scleral collagen fibres, are enough to visualise the intrascleral vessel in this region, it is not impossible to appreciate the $\mathrm{CZH}$ in FFA. Although some investigators have questioned the existence of this arterial circle in humans, ${ }^{2459}$ recent reports demonstrate the existence of the $\mathrm{CZH}$ by scanning electron microscopy performed on human cadaver eyes. ${ }^{6-810} 1213$ As far as we know, the exact FFA appearance of the arterial circle has not been reported to date.

We incidentally found an interesting vascular filling that was concentric to and in the vicinity of the temporal optic disc margin in fluorescein angiograms of myopic fundus 

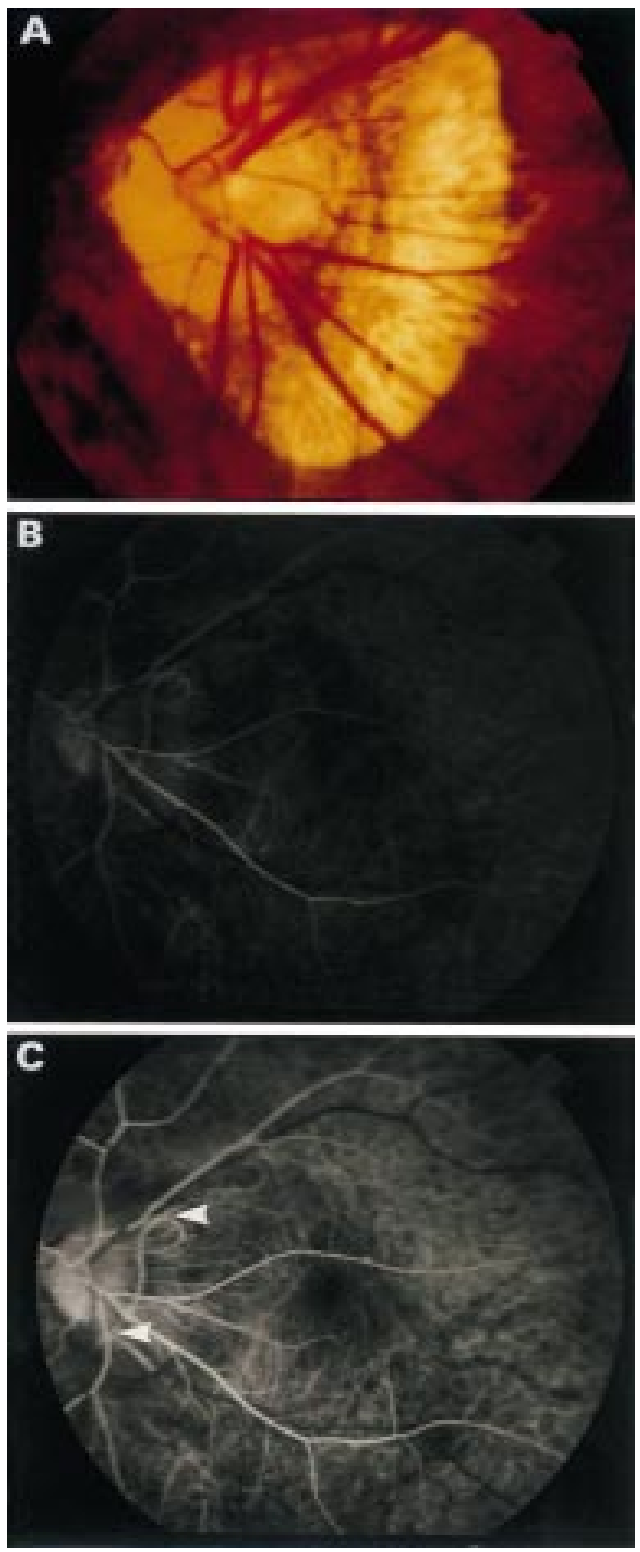

Figure 4 (A) Fundus photograph of the left eye of patient 4 with a refraction of -19.5 dioptres shows the concentric circle of Zinn-Haller within the temporal crescent. (B) The choroidal phase of fundus fuorescein angiography shows the vascular filling of the temporal part of the circle of Zinn-Haller. (C) The arterial phase of fundus fluorescein angiography shows the concentric shape of the circle of Zinn-Haller (between arrowheads) at the temporal aspect of the optic disc margin.

accompanied by a tilted disc (Fig 1). We suspected this vessel to be the temporal part of the $\mathrm{CZH}$ and investigated the FFA of patients with pathological myopia retrospectively to find out whether the arterial circle really could be observed in FFA and, if that was true, what its angiographic features were. We found a definite temporal part of the arterial $\mathrm{CZH}$ in only 15 of 307 eyes. However, that does not mean that these vessels are rare in humans because we excluded all ambiguous cases and insufficient myopic degeneration or some other cause might prevent the appearance of the $\mathrm{CZH}$ on FFA.

There have been many investigations on the microcirculation in the area of the optic nerve head and peripapillary choroid in humans and
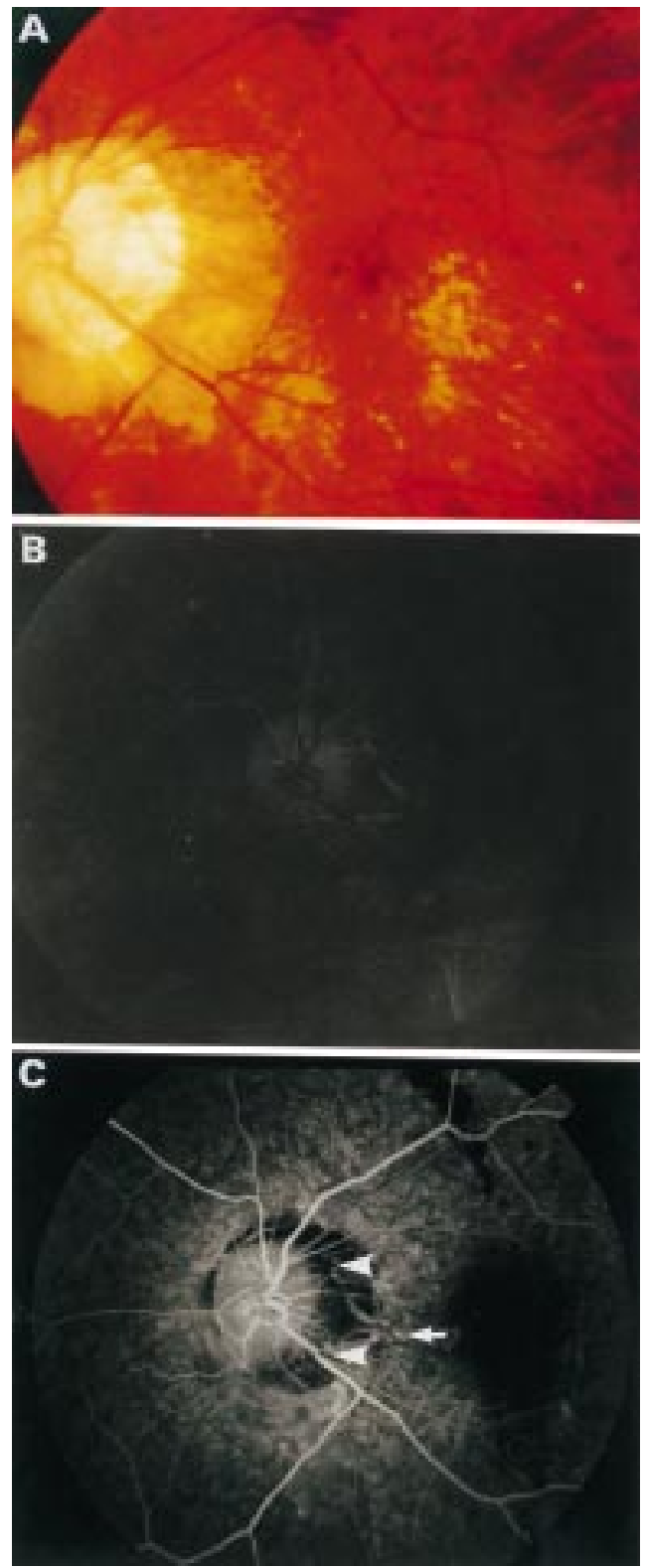

Figure 5 (A) Fundus photograph of the left eye of patient 6 with a refraction of -17.0 dioptres shows the temporal paraoptic short posterior ciliary artery beyond the temporal crescent margin. (B) The choroidal phase of fundus fluorescein angiography shows the vascular filling of the upper and lower arcades of the temporal paraoptic short posterior ciliary artery. (C) The venous phase of fundus fluorescein angiography shows the entry point of the temporal paraoptic short posterior ciliary artery (arrow) and its upper and lower divisions (between arrowheads).

primates using various techniques. ${ }^{2}$ 5-7 10 However, there have been various conclusions, especially in humans, because of interindividual variation of angioarchitecture and, more importantly, different examination methods. Hayreh $^{59}$ suggested that the $\mathrm{CZH}$ was rarely seen in humans and that it usually tends to be an incomplete circle when it is present. He also stressed the centripetal branches of the peripapillary choroidal arteries as a main source of blood supply to the prelaminar region of the optic nerve. In opposition to Hayreh's opinion, many authors have reported the frequent existence of the $\mathrm{CZH}$ and its major contribution to the circulation of the anterior part of the optic 


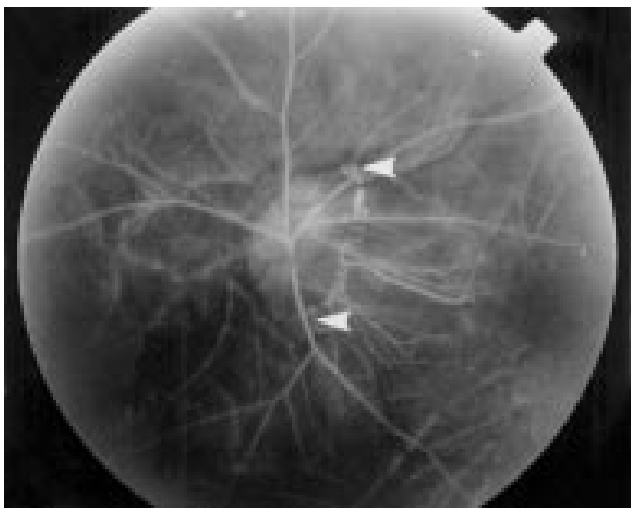

Figure 6 The left eye of patient 7 with a refraction of -21.5 dioptres. The early arteriovenous phase of fundus fluorescein angiography shows the concentric pattern of the temporal part of the circle of Zinn-Haller (between arrowheads) and tilted disc.
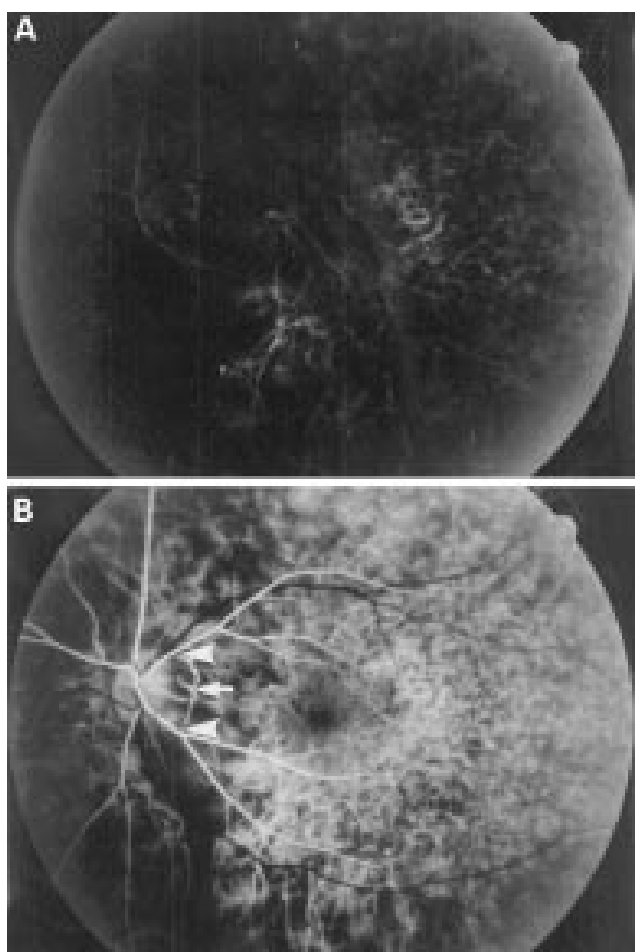

Figure 7 (A) The left eye of patient 9 with a refraction of -14.0 dioptres. The choroidal phase of fundus fluorescein angiography shows the vascular filling of the temporal part of the circle of Zinn-Haller. (B) The arterial phase of fundus fluorescein angiography shows the concentric shape of the circle of Zinn-Haller (between arrowheads) at the temporal aspect of the tilted disc. Note the entry point of the temporal paraoptic short posterior ciliary artery near the temporal optic disc margin (arrow).

nerve and peripapillary choroid under scanning electron microscope by using the microvascular corrosion castings in human cadaver eyes. ${ }^{671012} 13$ Olver et $a l^{712}$ observed the CZH in every specimen examined with vascular corrosion castings and they reported that the $\mathrm{CZH}$ was an elliptical circle divided into upper and lower arcades by the entry points of medial and lateral paraoptic SPCAs. They described the positional variation of the arterial circle in relation to the optic nerve, so it could lie near or at a distance from the optic nerve. Onda et $a l^{13}$ also demonstrated the existence of the perineural circular anastomosis approximately
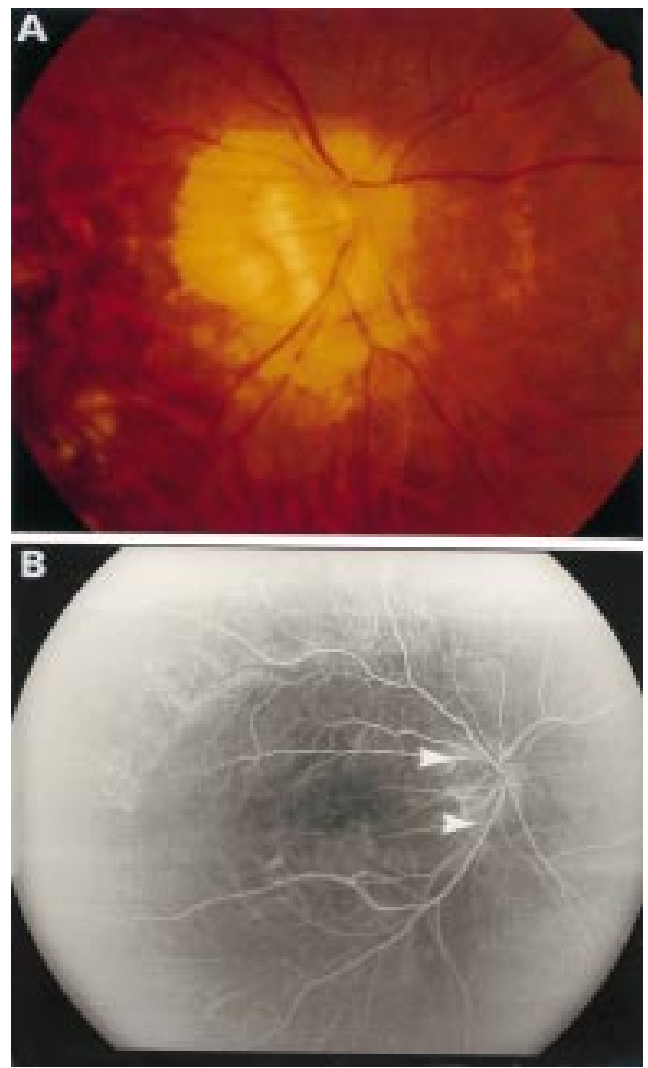

Figure 8 (A) Fundus photograph of the right eye of patient 10 with a refraction of -14.0 dioptres. (B) The venous phase of fundus fluorescein angiography shows the upper and lower arcades of the temporal part of the circle of Zinn-Haller (between arrowheads).

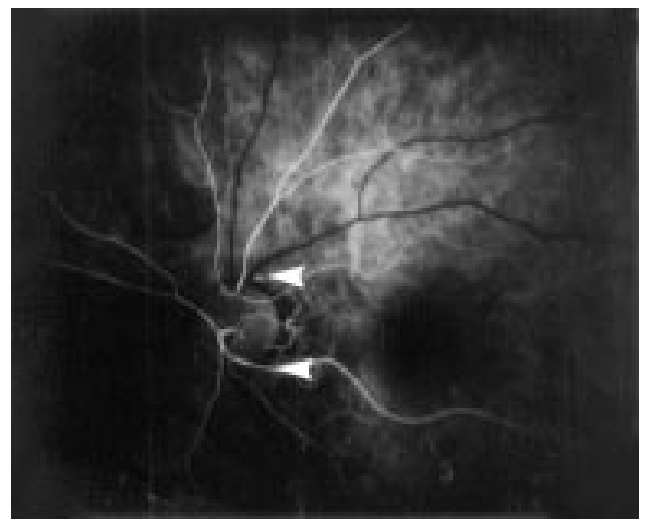

Figure 9 The left eye of patient 11 with a refraction of -15.0 dioptres. The arterial phase of fundus fluorescein angiography shows the zigzag shape of the temporal part of the circle of Zinn-Haller (between arrowheads).

200-300 $\mu \mathrm{m}$ posterior to the suprachoroidal space in 11 of 13 human eye bank eyes.

Because FFA may reveal only the part, especially the temporal part, of the circle in pathological myopia, our findings are limited to morphological and functional evaluation of the complete arterial circle. In this respect, indocyanine green angiography may have advantages over FFA because indocyanine green angiography can provide better visualisation not only of normal or pathological choroidal vasculature but also of retrobulbar vessels in pathological myopic eyes. ${ }^{14}$ However, our results suggest the variability of the $\mathrm{CZH}$ in 


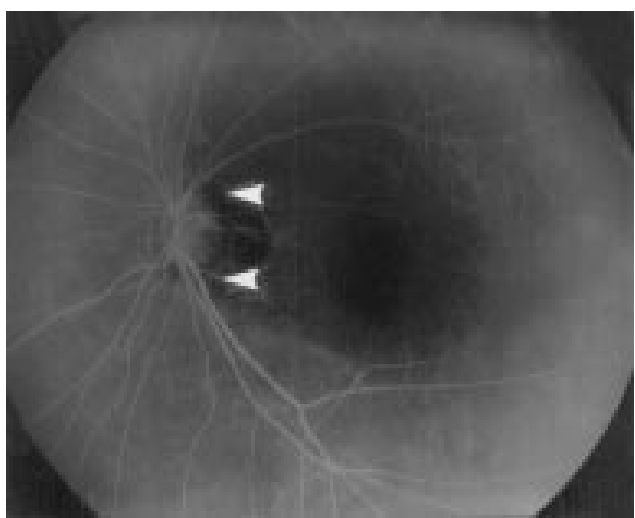

Figure 10 The left eye of patient 12 with a refraction of -8.50 dioptres. The venous phase of fundus fluorescein angiography shows the zigzag shape of the circle of Zinn-Haller (between arrowheads) within the temporal crescent.

location and shape. The positional variations of the circle relative to the optic nerve have already been documented. ${ }^{7}$ In many of our cases, the $\mathrm{CZH}$ is located closer to the optic disc margin than the temporal crescent margin although the location of the circle on FFA may be influenced by the degree of disc tilting or the amount of myopic crescent. We classify the shape or pattern of the $\mathrm{CZH}$ into a concentric and a zigzag pattern. Further investigation will be necessary to clarify the clinical implications of this morphological variations of the $\mathrm{CZH}$. Here we speculate that the circulatory disturbance of the optic nerve head may be related to the shape of the circle-namely, the concentric circle will have less chance of insufficiency or obstruction than the zigzag pattern. Furthermore, the distance between the temporal optic disc margin and the entry point of the temporal paraoptic SPCA also shows variation. As shown in patients 6 and 9, the entry point could be observed near the optic disc margin (Fig 7) or beyond the border of the temporal crescent (Fig 5). Although the above mentioned variations of the $\mathrm{CZH}$ may not be representative of the complete circle, we speculate that the invisible portion of the circle may have similar configurations as that of the visible counter portion.

According to an anatomical study, the completeness of the $\mathrm{CZH}$ was about $77 \%$ though this did not mean its functional completeness. ${ }^{12}$ In our cases, the completeness of the $\mathrm{CZH}$ cannot be evaluated. However, the visible parts of the arterial circles were continuous in their course, so it might be possible that the invisible parts of the circles are continuous. Therefore, the entire circle could be a complete one without interruption.

Detailed knowledge of the vascular system supplying the anterior optic nerve may be essential to understand the possible role of ischaemia in diseases such as anterior ischaemic optic neuropathy, glaucomatous disc cupping, and other related systemic diseases. Therefore, the angiographic appearance of the arterial $\mathrm{CZH}$ has some clinical importance though FFA has limitations in identifying the complete vasculature. In conclusion, the temporal part of the $\mathrm{CZH}$ can be observed by FFA in high myopia with a tilted disc. It is typically located within the temporal crescent and has morphological variations. It may be impossible to find 360 degrees of the CZH on FFA. However, if sufficient chorioretinal degeneration and scleral thinning extend to the nasal side of the optic nerve, we may have a chance of seeing the entire circle on FFA.

Presented at the Association for Research in Vision and Ophthalmology (ARVO), 21-26 April 1996, Fort Lauderdale, Florida, USA

1 Henkind P, Levitzky M. Angioarchitecture of the optic nerve, I: the papilla. Am f Ophthalmol 1969;68:979-86.

2 Levitzky M, Henkind P. Angioarchitecture of the optic nevitzy M, II: lamina cribrosa. Am f Ophthalmol 1969;68:986-
nerve 96.

3 Anderson DR, Braverman S. Reevaluation of the optic disc vasculature. Am f Ophthalmol 1976;82:165-74.

4 Leiberman MF, Maumenee AE, Green WR. Histologic studies of the vasculature of the anterior optic nerve. Am $\mathcal{F}$ Ophthalmol 1976;82:405-23.

5 Hayreh SS. Blood supply of the optic nerve head and its role in optic atrophy, glaucoma, and oedema of the optic nerve. Br f Ophthalmol 1969;53:721-48.

6 Fryczkowski AW, Grimson BS, Peiffer RL, Jr. Scanning electron microscopy of vascular casts of the human scleral lamina cribrosa. Int Ophthalmol 1984;7:95-100.

7 Olver JM, Spalton DJ, McCartney ACE. Microvascular study of the retrolaminar optic nerve in man: the possible significance in anterior ischaemic optic neuropathy. Eye 1990;4:7-24.

8 Olver JM. Functional anatomy of the choroidal circulation: methyl methacrylate casting of human choroid. Eye methyl methacry

9 Hayreh SS. Blood supply and vascular disorders of the optic nerve. An Inst Barraquer 1963;4:7-109.

10 Zhao Y, Li F. Microangioarchitecture of optic papilla. $f p n \mathcal{F}$ Ophthalmol 1987;31:147-59.

11 Anderson DR. Vascular supply to the optic nerve of primates. Am f Ophthalmol 1970;70:341-51.

12 Olver JM, Spalton DJ, McCartney ACE. Quantitative morphology of human retrolaminar optic nerve vasculature. Invest Ophthalmol Vis Sci 1994;35:3858-66.

13 Onda E, Cioffi GA, Bacon DR, Buskirk MV. Microvasculature of the human optic nerve. Am f Ophthalmol 1995;120: $92-102$.

14 Quaranta M, Arnold J, Coscas G, Francais C, Quentel G, Kuhn D, et al. Indocyanine green angiographic features of pathologic myopia. Am $\mathcal{F}$ Ophthalmol 1996;122:663-71. 